rather than boundary violations. I personally do not believe that this artificial distinction actually adds anything to the discourse, a discourse based on the timeless notion of trust in the profession (Sarkar 2004). More than the actual act, it is the degree of exploitation, not as felt by the therapist necessarily but as perceived by others (including the patient), that essentially influences outcomes.

Dr McQueen very correctly brings the concept of attachment into the mix. Although schools of psychotherapy are divided on how much 'transference' is responsible for therapy, the concept of attachment is less contentious. Attachment is all pervasive, and healthy attachment is necessary not only in therapy but in general development. Research is emerging on the fact that early attachment behaviour is replicated in adult behaviour, including attachment behaviour as adults. This could be attachment to partners, children or therapists. Attachment behaviour is central to interpersonal relationships, of which the therapist-patient relationship is but one. Whether one calls it transference or attachment matters little as long as it is agreed that it is the dynamic of a therapeutic dyad that is perhaps curative or facilitatory in treatment. The scenarios I discussed in my original article (Sarkar 2004) have dysfunctional attachment (on the part of both the therapist and the patient) at their core, which may manifest as abuse of transference or merely abuse of one's position as a doctor.

Gutheil TG, Gabbard G0 (1993) The concept of boundaries in clinical practice: theoretical and risk management dimensions. American Journal of Psychiatry; 150: 188-96.

Sarkar SP (2004) Boundary violation and sexual exploitation in psychiatry and psychotherapy: a review. Advances in Psychiatric Treatment; 10: 312-20.

Sameer P. Sarkar Forensic and General Psychiatrist, PO Box 3544, Wokingham, Berkshire RG40 9FA, UK. Email: spsarkar@ onetel.com

doi: 10.1192/apt.15.4.318b

\section{Antidepressants and bleeding}

In Palaniyappan et al's (2009) useful article, the side-effects of combination treatments neatly follow the description of each combination, but none mentions bleeding. Abnormal bleeding with selective serotonin reuptake inhibitors (SSRIs) has been studied and reviewed. One of the authors (Nick Ferrier) has written on the subject and recommended caution when prescribing SSRIs to patients at risk of gastrointestinal bleeds in particular (Paton 2005).

Palaniyappan et al note that the best-evidenced combination antidepressant treatment is an SSRI plus either a noradrenergic and specific serotonergic antidepressant (NaSSA) or trazodone. Meijer et al (2004) have demonstrated that the degree of inhibition of serotonin reuptake is associated with risk of abnormal bleeding. Furthermore, de Abajo et al (1999) found that the highest association between antidepressant use and gastrointestinal bleeds occurs with trazodone (despite it being a weak serotonin reuptake inhibitor). It may be reasonable, therefore, to expect the combination of an SSRI and trazodone, through differing mechanisms, to be associated with significant abnormal bleeding.

de Abajo FJ, Rodríguez LAG, Montero D (1999) Association between selective serotonin reuptake inhibitors and upper gastrointestinal bleeding: population based case control study. BMJ; 319: 1106-9.

Meijer WEE, Heerdink ER, Nolen WA, et al (2004) Association of risk of abnormal bleeding with degree of serotonin reuptake inhibition by antidepressants. Archives of Internal Medicine; 164: 2367-70.

Palaniyappan L, Insole L, Ferrier N (2009) Combining antidepressants: a review of evidence. Advances in Psychiatric Treatment, 15: 90-9.

Paton C, Ferrier IN (2005) SSRIs and gastrointestinal bleeding. BMJ; 331: $529-30$

Andrew Al-Adwani Consultant psychiatrist, Great Oaks, Ashby High Street, Ashby, North Lincolnshire DN16 2JX, UK. Email:

al-adwani@ntlworld.com

doi: 10.1192/apt.15.4.319 